\title{
PULMONARY FUNCTION IN SARCOIDOSIS
}

BY

\author{
R. MARSHALL, ${ }^{*}$ HUGH SMELLIE, J. H. BAYLIS, ClIFFORD HOYLE, AND D. V. BATES $\dagger$ \\ From the Medical Unit, St. Bartholomew's Hospital, London, and King's College Hospital, London
}

(RECEIVED FOR PUBLICATION JANUARY 13, 1958)

The effects of sarcoidosis upon lung function have already been studied by a number of investigators. Usually, however, sarcoidosis has been included only as one of a group of diseases producing pulmonary fibrosis, and sarcoidosis itself has not been specifically investigated. The purpose of this investigation was to study pulmonary function in sarcoidosis at all stages and to determine the nature of any disturbance and changes associated with progression of the disease. The abnormalities of function associated with particular radiographic appearances were also investigated, and we tried to assess the value of the various tests of function in gauging disability, progress, and prognosis.

\section{METHODS}

In order to study the disease at different stages the patients were chosen for investigation from a series of 200 according to the duration and radiographic form of their disease. They were allocated accordingly to one of five groups. These groups were :

Group I.-Patients with long-standing pulmonary fibrosis as shown radiographically by hilar distortion, often with elevation, and linear shadows.

GrouP II.-Patients with infiltrative shadows present for more than two years.

Group III.-Patients with infiltrative shadows known to have been present for less than two years since diagnosis.

GrouP IV.-Patients known to have been in Groups II or III but in whom radiologically the lung fields had cleared completely.

Group V.-Patients with hilar lymph node enlargement only and normal lungs radiographically.

Twenty-six patients (11 men and 15 women) were investigated: of these, six were in Group I, three in Group II, six in Group III, six in Group

\footnotetext{
* Present address: Nuffield Department of Surgery, Radcliffe Infirmary, Oxford.

+Present address: Royal Victoria Hospital, Montreal, Canada.
}

IV, and five in Group V. The diagnosis of sarcoidosis was supported by biopsy of either liver, lymph node, skin, or conjunctiva in 20 of the 26 patients. In four others liver biopsy was negative and in two was not attempted. Most patients had previously been treated with streptomycin and P.A.S. and nine had received cortisone (Table I). We excluded patients with cardiac disease or pulmonary disease other than sarcoidosis and the one patient with dyspnoea more severe than grade 3 (see classification below) who was unsuitable for exercise tests. The clinical category of the patients and the clinician's assessment of dyspnoea were not known to the observers carrying out the pulmonary function tests until after these had been assessed.

A clinical assessment of dyspnoea was made from the history of exercise tolerance according to the following grades:

Grade 0.-Normal performance without dyspnoea for a person of comparable age.

Grade 1.-Dyspnoea on moderate effort, i.e., running for a bus or inability to climb stairs or hills quickly.

Grade 2.-Can walk on the level without dyspnoea but additional tasks not possible. Can climb one flight of stairs without undue distress.

Grade 3.-Can walk up to 500 yards on level without stopping. Capable of sedentary work and light household duties.

\section{Pulmonary Function Tests}

The measurements of pulmonary function made on these patients were (a) the subdivisions of lung volume, $(b)$ the mixing efficiency of the lungs, (c) the maximum breathing capacity (M.B.C.), (d) the fast expiratory flow rate (E.F.R. $\left.{ }^{40}\right),(e)$ the diffusing capacity for carbon monoxide $\left(D_{00}\right)$, $(f)$ the lung compliance, and $(g)$ the non-elastic resistance of the lungs.

The subdivisions of lung volume have been named according to the standard terminology (Pappenheimer, Comroe, Cournand, Ferguson, 
TABLE I

CLINICAL DATA ON 26 PATIENTS WITH PULMONARY SARCOIDOSIS

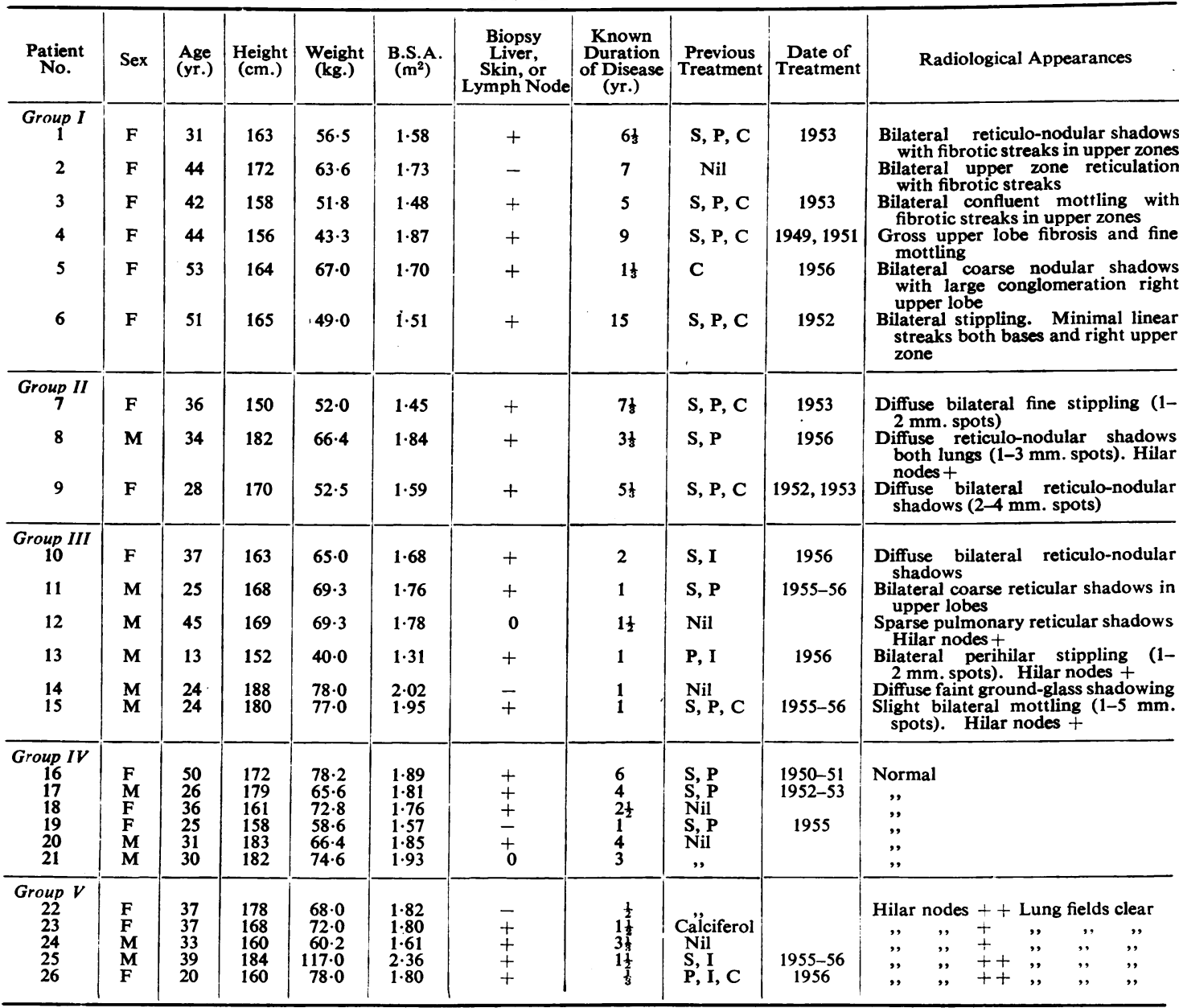

$\mathbf{S}=$ Str eptomycin. $\mathbf{P}=$ P.A.S. $\mathbf{C}=$ Cortisone. $\mathbf{I}=$ Isoniazid.

Filley, Fowler, Gray, Helmholtz, Otis, Rahn, and Riley, 1950 ; Comroe, Forster, DuBois, Briscoe, and Carlsen, 1955). The mixing efficiency of the lungs is predominantly a measure of the uniformity of alveolar ventilation. The fast expiratory flow rate is the maximum rate of air flow which is attained when a subject makes a forced expiration from the full inspiratory position. This was measured in addition to the M.B.C., because, although the latter is more familiar to clinicians, the former gives more consistent results, is easier to perform, and is not so fatiguing to the patient. The diffusing capacity of the lungs is a measure of the quantity of a gas, such as oxygen, which can pass per minute from the alveoli into the blood with a pressure gradient of
$1 \mathrm{~mm}$. Hg. The diffusing capacity for carbon monoxide has been measured, since carbon monoxide behaves similarly to oxygen and the method is technically easier. The lung compliance is a measure of the elastic properties of the lung and is expressed as the increase in lung volume (in litres) per centimetre water increase in the pressure distending the lung. The non-elastic resistance which has been measured is the total frictional resistance to lung movement; this includes both the resistance to flow of air through the airways and the frictional resistances of the lung tissues themselves. In this study it was not possible to measure the two factors separately, but previous work (Marshall and DuBois, 1956) has shown that in sarcoidosis the tissue resistance 
factor may be the major component of the nonelastic resistance.

The pulmonary function tests were summarized for each patient by separate assessment of ventilatory defect, diffusion defect, and compliance defect, these assessments being made on the results of the expiratory flow rate, diffusing capacity for carbon monoxide, and compliance measurements respectively. Each function was assessed on the basis of four grades and points were allotted according to the following scale: normal, 0 ; slightly reduced, 1 ; moderately reduced, 2 ; and severely reduced, 3. The patient's F.R.C. was taken into consideration in the assessment of both the diffusing capacity (Bates, Boucot, and Dormer, 1955) and compliance (Marshall, 1957). The normality of the $D_{c o}$ was assessed on the exercise figure. The compliance was assessed from the resting figures only, since in these patients there was usually no appreciable change on exercise. A major difficulty arises in deciding whether particular values of the diffusing capacity, and the compliance are normal, namely, should these values be compared to normal values predicted from the surface area of the subject, or to values predicted for a normal subject of similar lung volume? In analysing the results the latter method has been followed, as this seems a more practical way of assessing the changes in this particular condition.

TECHNIQUES.-All the pulmonary function tests on each patient were carried out on the same day. The subdivisions of the vital capacity were measured using a closed circuit spirometer and with the patient seated. The functional residual capacity (F.R.C.) and mixing efficiency (M.E.\%) were measured by the closed circuit helium method described by Bates and Christie (1950) and the index used here is independent of tidal volume and lung volume. The values obtained for lung volumes were corrected to body temperature and pressure saturated. The predicted values for lung volumes shown in Table II were calculated from the formulae derived by Needham, Rogan, and McDonald (1954). All the predicted lung volumes except for the F.R.C. in adults were calculated from the simplified regression equations. The predicted F.R.C. in adults was calculated from the "best regression equation" (Table $X$ of Needham and others). The comparisons between these predicted values and the lung volumes found is believed to be valid, since identical methods of measurement have been used, and the range and means of values found in normal subjects by Needham and others and by ourselves have been comparable.

The maximum breathing capacity (M.B.C.) was measured by breathing through a low-resistance valve from a bag containing $5 \% \mathrm{CO}_{2}$ in oxygen. The volume removed from the bag during 15 seconds of maximum ventilation was measured, and this volume, multiplied by 4 , is given as the M.B.C. The expiratory flow rate (E.F.R. ${ }^{40}$ ) was measured as described by Kennedy (1953) but using a spirometer with a light-weight bell. The diffusing capacity for carbon monoxide was measured by the method described by Bates and others (1955) and calculated by their second method $\left(D_{c o} I I\right)$ which assumes that the end tidal sample represents the mean alveolar tension. The lung compliance and non-elastic resistance were measured by simultaneous measurement of intraoesophageal pressure and air flow or tidal volume. The technique used for measurement of compliance was that described by Marshall and DuBois (1956) and the non-elastic resistance was measured by the subtraction method of Mead and Whittenberger (1953).

Measurements of diffusing capacity and lung mechanics were made with the patient seated at rest and were then repeated with the patient exercising on a motor-driven treadmill. No attempt was made to exercise the patients to their limits of tolerance, and the treadmill speed was one that the patient could maintain for about 10 minutes without undue discomfort. The "experimental" assessment of dyspnoea was made by two observers (R. M. and D. V. B.) on the patients' performance on the treadmill, and on whether the patient was actually dyspnoeic at the end of the exercise period. At the time this assessment was made, none of the results of the function tests had been calculated.

\section{RESULTS}

The results obtained from measurement of lung volumes are shown in Table II. All the lung volumes are, in general, slightly reduced and this reduction is greater in Groups I, II, and III, where there is more radiographic change in the lungs than in Groups IV and V. In each group, however, there is a large variation, and, in particular in patient 23 in Group V, lung volumes are grossly reduced. The mixing efficiency is normal in most of the subjects.

The diffusing capacity on exercise assessed in relation to the patients' F.R.C. is shown in Fig. 1. Impairment of diffusing capacity is more marked in patients in Groups I and II (Table III) although two patients in Group I have a normal diffusing capacity. Five patients in Group III have a normal $\mathbf{D}_{\mathrm{co}}$ in spite of radiographic changes in the lung. On the other hand, five out of six patients in Group IV have a diffusion defect although the lung fields are now normal radiographically. The patients in Group $V$ who have never had any evidence of lung involvement have, with one exception, normal diffusing capacities.

The pulmonary compliance, when assessed in relation to the patient's F.R.C., is surprisingly normal in most patients and only lies below the lower limit of normal in four patients, two in 
TABLE II

LUNG VOLUMES, M.B.C., AND E.F.R. ${ }^{40}$ IN 26 PATIENTS WITH PULMONARY SARCOIDOSIS

\begin{tabular}{|c|c|c|c|c|c|c|c|c|c|c|c|c|c|c|}
\hline \multirow{2}{*}{$\begin{array}{l}\text { Patient } \\
\text { No. }\end{array}$} & \multicolumn{2}{|c|}{ V.C. (1.) } & \multicolumn{2}{|c|}{ I.C. (1.) } & \multicolumn{2}{|c|}{ E.R.V. (1.) } & \multicolumn{2}{|c|}{ F.R.C. (1.) } & \multicolumn{2}{|c|}{ R.V. (1.) } & \multirow{2}{*}{$\frac{\text { R.V. }}{\text { T.L.C. }}(\%)$} & \multirow{2}{*}{$\begin{array}{l}\text { M.E. } \\
(\%)\end{array}$} & \multirow{2}{*}{$\begin{array}{c}\text { M.B.C. } \\
\text { (1.i. } \\
\text { in.) }\end{array}$} & \multirow{2}{*}{$\begin{array}{l}\text { E.F.R. }{ }^{40} \\
\text { (1./min.) }\end{array}$} \\
\hline & Observed & $\%$ Pred. & Observed & $\%$ Pred. & Observed & $\%$ Pred. & Observed & $\%$ Pred. & Observed & $\%$ Pred. & & & & \\
\hline $\begin{array}{c}\text { Group I } \\
1 \\
2 \\
3 \\
4 \\
5 \\
6 \\
\end{array}$ & $\begin{array}{l}3 \cdot 04 \\
2 \cdot 77 \\
2 \cdot 20 \\
1.41 \\
2 \cdot 38 \\
2 \cdot 66\end{array}$ & $\begin{array}{l}99 \\
89 \\
83 \\
56 \\
91 \\
96\end{array}$ & $\begin{array}{l}2.12 \\
1.93 \\
1.46 \\
0.92 \\
2.02 \\
1.33\end{array}$ & $\begin{array}{r}96 \\
80 \\
76 \\
51 \\
100 \\
62\end{array}$ & $\begin{array}{l}0.92 \\
0.84 \\
0.74 \\
0.49 \\
0.36 \\
1.33\end{array}$ & $\begin{array}{r}108 \\
118 \\
101 \\
67 \\
59 \\
211\end{array}$ & $\begin{array}{l}2 \cdot 34 \\
2 \cdot 12 \\
2 \cdot 39 \\
1.76 \\
1.65 \\
3 \cdot 36\end{array}$ & $\begin{array}{r}97 \\
84 \\
101 \\
74 \\
79 \\
124\end{array}$ & $\begin{array}{l}1.42 \\
1.34 \\
1.65 \\
1.27 \\
1.29 \\
2.03\end{array}$ & $\begin{array}{r}96 \\
84 \\
104 \\
79 \\
77 \\
122\end{array}$ & $\begin{array}{l}32 \\
33 \\
43 \\
47 \\
35 \\
43\end{array}$ & $\begin{array}{l}60 \\
73 \\
72 \\
43 \\
57 \\
55\end{array}$ & $\begin{array}{l}89 \\
70 \\
78 \\
31 \\
28 \\
48\end{array}$ & $\begin{array}{l}88 \\
73 \\
93 \\
30 \\
62 \\
94\end{array}$ \\
\hline $\begin{array}{c}\text { Group } I I \\
7 \\
8 \\
9 \\
\end{array}$ & $\begin{array}{l}1.93 \\
4.09 \\
2.42 \\
\end{array}$ & $\begin{array}{l}78 \\
87 \\
71 \\
\end{array}$ & $\begin{array}{l}1.28 \\
2.92 \\
1.77 \\
\end{array}$ & $\begin{array}{l}76 \\
87 \\
70 \\
\end{array}$ & $\begin{array}{l}0.65 \\
1.17 \\
0.65\end{array}$ & $\begin{array}{l}82 \\
86 \\
74 \\
\end{array}$ & $\begin{array}{l}1 \cdot 36 \\
3 \cdot 06 \\
2 \cdot 32 \\
\end{array}$ & $\begin{array}{l}63 \\
72 \\
82 \\
\end{array}$ & $\begin{array}{l}0.71 \\
1.89 \\
1.67 \\
\end{array}$ & $\begin{array}{r}47 \\
96 \\
115 \\
\end{array}$ & $\begin{array}{l}27 \\
32 \\
41 \\
\end{array}$ & $\begin{array}{l}40 \\
57 \\
45 \\
\end{array}$ & $\begin{array}{r}73 \\
97 \\
54 \\
\end{array}$ & $\begin{array}{l}60 \\
94 \\
75 \\
\end{array}$ \\
\hline $\begin{array}{c}\text { Group III } \\
10 \\
11 \\
12 \\
13 \\
14 \\
15\end{array}$ & $\begin{array}{l}2.03 \\
4.05 \\
3.58 \\
2.14 \\
5 \cdot 18 \\
4 \cdot 26\end{array}$ & $\begin{array}{l}69 \\
85 \\
95 \\
78 \\
96 \\
84\end{array}$ & $\begin{array}{l}1.65 \\
3.05 \\
2.51 \\
1.57 \\
3.65 \\
3.33\end{array}$ & $\begin{array}{r}76 \\
103 \\
70 \\
81 \\
94 \\
94\end{array}$ & $\begin{array}{l}0.38 \\
1.00 \\
1.07 \\
0.59 \\
1.53 \\
0.93\end{array}$ & $\begin{array}{r}49 \\
67 \\
92 \\
73 \\
101 \\
61\end{array}$ & $\begin{array}{l}1.52 \\
2.54 \\
2.10 \\
1.16 \\
4.69 \\
2.74\end{array}$ & $\begin{array}{r}71 \\
90 \\
69 \\
67 \\
120 \\
80\end{array}$ & $\begin{array}{l}1.14 \\
1.54 \\
1.03 \\
0.57 \\
3.16 \\
1.81\end{array}$ & $\begin{array}{r}75 \\
86 \\
47 \\
62 \\
177 \\
101\end{array}$ & $\begin{array}{l}36 \\
28 \\
22 \\
21 \\
38 \\
30\end{array}$ & $\begin{array}{l}31 \\
81 \\
51 \\
77 \\
52 \\
52\end{array}$ & $\begin{array}{r}76 \\
128 \\
114 \\
63 \\
160 \\
102\end{array}$ & $\begin{array}{r}82 \\
151 \\
112 \\
72 \\
151 \\
121\end{array}$ \\
\hline $\begin{array}{c}\text { Group IV } \\
16 \\
17 \\
18 \\
19 \\
20 \\
21\end{array}$ & $\begin{array}{l}2 \cdot 60 \\
4 \cdot 21 \\
3 \cdot 28 \\
2 \cdot 72 \\
5 \cdot 02 \\
4 \cdot 29\end{array}$ & $\begin{array}{r}67 \\
86 \\
114 \\
91 \\
102 \\
88\end{array}$ & $\begin{array}{l}1.57 \\
2.63 \\
2.28 \\
2.02 \\
3.25 \\
2.95\end{array}$ & $\begin{array}{r}67 \\
78 \\
110 \\
97 \\
92 \\
86\end{array}$ & $\begin{array}{l}0.65 \\
1.58 \\
1.00 \\
0.70 \\
1.77 \\
1.34\end{array}$ & $\begin{array}{r}102 \\
106 \\
127 \\
77 \\
126 \\
94\end{array}$ & $\begin{array}{l}1.86 \\
3.45 \\
2.42 \\
1.96 \\
4.06 \\
3.73\end{array}$ & $\begin{array}{r}91 \\
92 \\
132 \\
91 \\
100 \\
106\end{array}$ & $\begin{array}{l}1.21 \\
1.87 \\
1.42 \\
1.26 \\
2.29 \\
2.39\end{array}$ & $\begin{array}{r}73 \\
103 \\
93 \\
89 \\
119 \\
126\end{array}$ & $\begin{array}{l}35 \\
31 \\
30 \\
32 \\
31 \\
36\end{array}$ & $\begin{array}{l}60 \\
78 \\
68 \\
57 \\
70 \\
72\end{array}$ & $\begin{array}{r}32 \\
151 \\
49 \\
69 \\
141 \\
131\end{array}$ & $\begin{array}{r}75 \\
160 \\
92 \\
80 \\
168 \\
165\end{array}$ \\
\hline $\begin{array}{c}\text { Group } V \\
22 \\
23 \\
24 \\
25 \\
26\end{array}$ & \begin{tabular}{|l|}
$2 \cdot 92$ \\
$1 \cdot 55$ \\
$3 \cdot 46$ \\
$4 \cdot 30$ \\
$3 \cdot 19$ \\
\end{tabular} & $\begin{array}{l}82 \\
49 \\
90 \\
93 \\
90\end{array}$ & $\begin{array}{l}1.96 \\
1 \cdot 12 \\
2.02 \\
3.48 \\
2 \cdot 23\end{array}$ & $\begin{array}{r}71 \\
47 \\
81 \\
103 \\
100\end{array}$ & $\begin{array}{l}0.96 \\
0.43 \\
1.44 \\
0.82 \\
0.63\end{array}$ & $\begin{array}{r}123 \\
55 \\
105 \\
65 \\
65\end{array}$ & $\begin{array}{l}2.63 \\
1.44 \\
2.80 \\
2.00 \\
1.47\end{array}$ & $\begin{array}{r}101 \\
67 \\
103 \\
93 \\
90\end{array}$ & $\begin{array}{l}1.67 \\
1.01 \\
1.36 \\
1.18 \\
0.84\end{array}$ & $\begin{array}{r}109 \\
66 \\
69 \\
57 \\
62\end{array}$ & $\begin{array}{l}36 \\
39 \\
28 \\
22 \\
23\end{array}$ & $\begin{array}{l}72 \\
53 \\
96 \\
68 \\
72\end{array}$ & $\begin{array}{l}71 \\
63 \\
87 \\
95 \\
58\end{array}$ & $\begin{array}{r}50 \\
56 \\
98 \\
119 \\
102\end{array}$ \\
\hline
\end{tabular}

V.C. = Vital capacity. I.C. = Inspiratory capacity. E.R.V. = Expiratory reserve volume. F.R.C. = Functional residual capacity. R.V. $=$ Residual volume. T.L.C. $=$ Total lung capacity. Obs. $=$ Observed value. \% Pred. $=$ Observed values as a percentage of predicted value. M.E. $\%=$ Mixing efficiency percentage of normal. M.B.C. $=$ Maximum breathing capacity. E.F.R. ${ }^{40}=$ Expiratory flow rate (Kennedy, 1953).

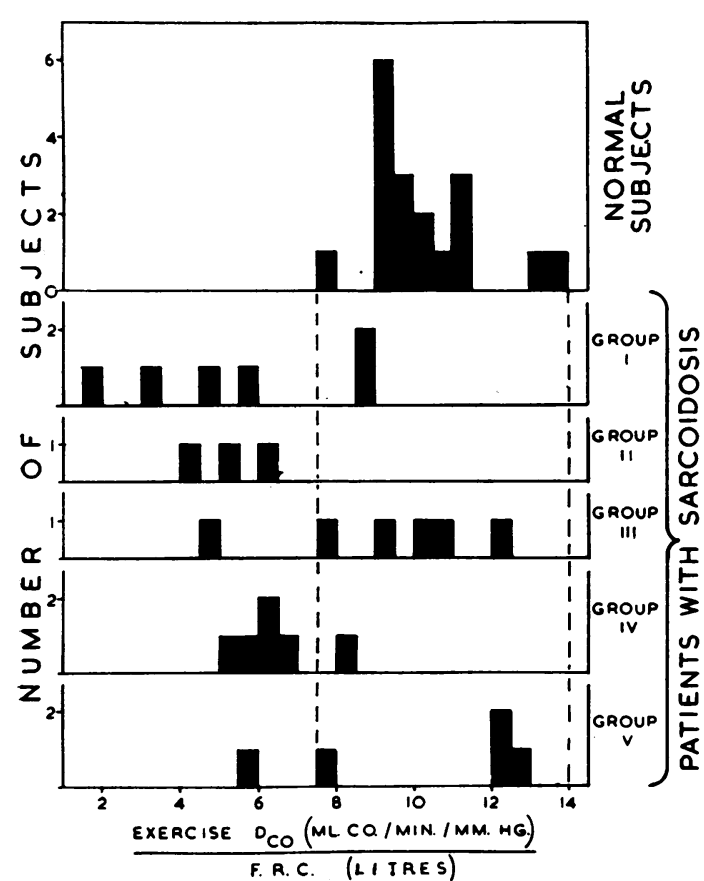

FIG. 1.-Histogram of Dco'F.R.C. in the patients with sarcoidosis compared with the values obtained in 18 normal subjects.

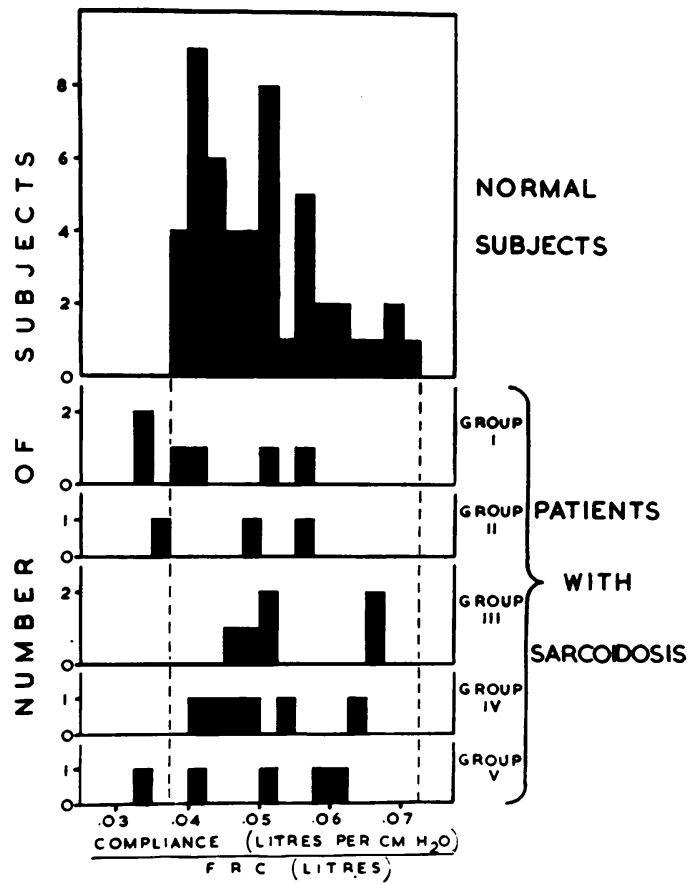

FIG. 2.-Histogram of compliance/F.R.C. in the patients with sarcoidosis compared with the values obtained in $\mathbf{5 0}$ normal subjects. 
TABLE III

DIFFUSING CAPACITY FOR CARBON MONOXIDE AND LUNG MECHANICS IN 26 PATIENTS WITH SARCOIDOSIS AT REST AND ON EXERCISE

\begin{tabular}{|c|c|c|c|c|c|c|c|c|c|c|c|c|c|}
\hline \multirow[b]{2}{*}{$\begin{array}{l}\text { Patient } \\
\text { No. }\end{array}$} & \multirow[b]{2}{*}{$\begin{array}{c}\text { Minute } \\
\text { Volume } \\
\text { (1./min.) }\end{array}$} & \multicolumn{4}{|c|}{ Rest } & \multicolumn{7}{|c|}{ Exercise } & \multirow[b]{2}{*}{$\begin{array}{l}\text { Non-elasti } \\
\text { Resistance } \\
\left(\mathrm{cm} . \mathrm{H}_{2} \mathrm{O}\right. \\
1 . / \mathrm{sec} \text { at } \\
\left.0.51 . \text { sec. }^{\prime}\right)\end{array}$} \\
\hline & & $\begin{array}{c}\text { Res- } \\
\text { pirations } \\
\text { per } \\
\text { Minute }\end{array}$ & $\begin{array}{c}\mathrm{D}_{\mathrm{co}} \\
(\mathrm{ml} . / \mathrm{min} . \\
\mathrm{mm} . \mathrm{Hg})\end{array}$ & $\begin{array}{c}\text { Com- } \\
\text { pliance } \\
(1 . / \mathrm{cm} . \\
\left.\mathrm{H}_{2} \mathrm{O}\right)\end{array}$ & $\mid \begin{array}{l}\text { Non-elastic } \\
\text { Resistance } \\
\left(\mathrm{cm} . \mathrm{H}_{2} \mathrm{O} /\right. \\
1 . / \mathrm{sec} . \text { at } \\
0.51 . / \mathrm{sec} .)\end{array}$ & $\begin{array}{c}\text { Tread- } \\
\text { mill } \\
\text { Speed } \\
\text { (m.p.h.) }\end{array}$ & $\begin{array}{l}\text { Minute } \\
\text { Volume } \\
(1 . / \mathrm{min} .)\end{array}$ & $\begin{array}{c}\text { Res- } \\
\text { pirations } \\
\text { per } \\
\text { Minute }\end{array}$ & $\begin{array}{c}\mathrm{O}_{2} \\
\text { Uptake } \\
\text { (mi./ } \\
\text { min.) }\end{array}$ & $\frac{\mathrm{O}_{2}}{\mathrm{M} . \mathrm{V} .}$ & $\begin{array}{c}\mathbf{D}_{\mathrm{co}} \\
(\mathrm{ml} . \mathrm{min} . \\
\mathrm{mm} . \mathbf{H g})\end{array}$ & $\begin{array}{c}\text { Com- } \\
\text { pliance } \\
(1 . / \mathrm{cm} . \\
\left.\mathrm{H}_{2} \mathrm{O}\right)\end{array}$ & \\
\hline $\begin{array}{c}\text { Group I } \\
1 \\
2 \\
3 \\
4 \\
5 \\
6\end{array}$ & $\begin{array}{r}4.7 \\
11.4 \\
7.0 \\
9.4 \\
10.1 \\
13.7\end{array}$ & $\begin{array}{r}9 \\
20 \\
18 \\
18 \\
17 \\
31\end{array}$ & $\begin{array}{r}10.6 \\
13.0 \\
9.8 \\
3.8 \\
8.1 \\
4.1\end{array}$ & $\begin{array}{l}0.093 \\
0.118 \\
0.079 \\
0.071 \\
0.085 \\
0.116\end{array}$ & $\begin{array}{l}5.86 \\
3.62 \\
4.24 \\
8.04 \\
5.13 \\
1.92\end{array}$ & $\begin{array}{r}2 \frac{1}{2} \\
1 \\
1 \\
\frac{8}{4} \\
1 \frac{3}{4}\end{array}$ & $\begin{array}{l}12.8 \\
27.0 \\
13.8 \\
14.6 \\
13.8 \\
27.1\end{array}$ & $\begin{array}{l}10 \\
32 \\
25 \\
25 \\
19 \\
31\end{array}$ & $\begin{array}{l}740 \\
990 \\
660 \\
650 \\
780 \\
870\end{array}$ & $\begin{array}{l}5.78 \\
3.73 \\
4.74 \\
3.65 \\
4.70 \\
3.25\end{array}$ & $\begin{array}{r}20.9 \\
18.9 \\
11.9 \\
5.9 \\
8.7 \\
5.3\end{array}$ & $\begin{array}{l}0.101 \\
0.130 \\
0.096 \\
0.058 \\
0.087 \\
0.133\end{array}$ & $\begin{array}{l}4 \cdot 23 \\
2 \cdot 32 \\
2 \cdot 83 \\
6 \cdot 65 \\
3.96 \\
1.22\end{array}$ \\
\hline $\begin{array}{c}\text { Group } \text { II } \\
7 \\
8 \\
9\end{array}$ & $\begin{array}{l}9 \cdot 7 \\
8 \cdot 2 \\
8 \cdot 9\end{array}$ & $\begin{array}{l}17 \\
15 \\
20\end{array}$ & $\begin{array}{r}5 \cdot 5 \\
10 \cdot 6 \\
8 \cdot 2\end{array}$ & $\begin{array}{l}0 \cdot 051 \\
0 \cdot 147 \\
0 \cdot 125\end{array}$ & $\begin{array}{l}5 \cdot 04 \\
3 \cdot 17 \\
3 \cdot 11\end{array}$ & $\begin{array}{l}2 \\
2 \frac{1}{2}\end{array}$ & $\begin{array}{l}22.6 \\
19.0 \\
15 \cdot 4\end{array}$ & $\begin{array}{l}23 \\
16 \\
19\end{array}$ & $\begin{array}{r}390 \\
1,200 \\
590\end{array}$ & $\begin{array}{l}1.74 \\
6.30 \\
3.92\end{array}$ & $\begin{array}{r}6.0 \\
19 \cdot 5 \\
11 \cdot 8\end{array}$ & $\begin{array}{l}0.057 \\
0 \cdot 154 \\
0 \cdot 112\end{array}$ & $\begin{array}{l}2.00 \\
1.63 \\
2.76\end{array}$ \\
\hline $\begin{array}{c}\text { Group III } \\
10 \\
11 \\
12 \\
13 \\
14 \\
15\end{array}$ & $\begin{array}{r}5.7 \\
8.7 \\
15.8 \\
10.8 \\
18.2 \\
8.5\end{array}$ & $\begin{array}{l}14 \\
12 \\
23 \\
20 \\
17 \\
17\end{array}$ & $\begin{array}{l}10.0 \\
15.8 \\
11.2 \\
12.7 \\
14.4 \\
14.2\end{array}$ & $\begin{array}{l}0 \cdot 076 \\
0 \cdot 133 \\
0 \cdot 139 \\
0.078 \\
0 \cdot 217 \\
0 \cdot 143\end{array}$ & $\begin{array}{l}5.45 \\
2.93 \\
2.81 \\
4.39 \\
2.09 \\
2.19\end{array}$ & $\begin{array}{l}1 \frac{8}{4} \\
3 \\
2 \frac{1}{2} \\
1 \frac{1}{2} \\
3 \\
3\end{array}$ & $\begin{array}{l}12 \cdot 6 \\
16 \cdot 3 \\
24 \cdot 3 \\
20 \cdot 6 \\
28 \cdot 2 \\
22 \cdot 0\end{array}$ & $\begin{array}{l}14 \\
12 \\
22 \\
37 \\
13 \\
21\end{array}$ & $\begin{array}{r}760 \\
1,060 \\
1,220 \\
830 \\
1,600 \\
1,220\end{array}$ & $\begin{array}{l}6.00 \\
6.50 \\
5.05 \\
4.06 \\
5.65 \\
5.55\end{array}$ & $\begin{array}{l}18.4 \\
23.6 \\
16.5 \\
12.7 \\
21.4 \\
28.4\end{array}$ & $\begin{array}{l}0.078 \\
0.161 \\
0.200 \\
0.071 \\
0 \cdot 263 \\
0.178\end{array}$ & $\begin{array}{l}4.02 \\
2.08 \\
1.57 \\
2.49 \\
1.47 \\
1.62\end{array}$ \\
\hline $\begin{array}{c}\text { Group IV } \\
16 \\
17 \\
18 \\
19 \\
20 \\
21\end{array}$ & $\begin{array}{r}10.9 \\
8.6 \\
13.7 \\
7.0 \\
12.9 \\
13.2\end{array}$ & $\begin{array}{l}21 \\
17 \\
17 \\
20 \\
16 \\
16\end{array}$ & $\begin{array}{r}7.8 \\
9 \cdot 6 \\
12.9 \\
8.1 \\
17.9 \\
19 \cdot 2\end{array}$ & $\begin{array}{l}0 \cdot 120 \\
0 \cdot 149 \\
0 \cdot 115 \\
0 \cdot 094 \\
0 \cdot 222 \\
0 \cdot 152\end{array}$ & $\begin{array}{l}2.97 \\
2 \cdot 25 \\
3.70 \\
4 \cdot 21 \\
1.33 \\
2.09\end{array}$ & $\begin{array}{l}3^{\frac{3}{4}} \\
1 \\
\frac{1}{2} \\
3 \\
3\end{array}$ & $\begin{array}{l}20 \cdot 2 \\
22 \cdot 6 \\
18 \cdot 0 \\
14 \cdot 8 \\
27 \cdot 7 \\
23 \cdot 1\end{array}$ & $\begin{array}{l}24 \\
17 \\
15 \\
26 \\
13 \\
19\end{array}$ & $\begin{array}{r}890 \\
1,320 \\
830 \\
745 \\
1,360 \\
990\end{array}$ & $\begin{array}{l}4.41 \\
5.80 \\
4.62 \\
5.02 \\
4.92 \\
6.32\end{array}$ & $\begin{array}{l}10 \cdot 0 \\
20 \cdot 2 \\
16 \cdot 7 \\
12 \cdot 3 \\
34 \cdot 3 \\
24 \cdot 0\end{array}$ & $\begin{array}{l}0.119 \\
0.204 \\
0.161 \\
0.133 \\
0.238 \\
0.152\end{array}$ & $\begin{array}{l}2.56 \\
1.57 \\
2.93 \\
2.39 \\
1.39 \\
2.18\end{array}$ \\
\hline $\begin{array}{c}\text { Group } V \\
22 \\
23 \\
24 \\
25 \\
26\end{array}$ & $\begin{array}{r}7 \cdot 1 \\
9 \cdot 2 \\
7 \cdot 5 \\
12 \cdot 2 \\
6 \cdot 6\end{array}$ & $\begin{array}{l}18 \\
19 \\
11 \\
11 \\
10\end{array}$ & $\begin{array}{r}17 \cdot 0 \\
9 \cdot 7 \\
18 \cdot 2 \\
21 \cdot 8 \\
16.5\end{array}$ & $\begin{array}{l}0.164 \\
0.075 \\
0.096 \\
0.120 \\
0.061\end{array}$ & $\begin{array}{l}4 \cdot 28 \\
6 \cdot 75 \\
3 \cdot 39 \\
3 \cdot 34 \\
3 \cdot 86\end{array}$ & $\begin{array}{l}1 \\
1 \\
2 \frac{1}{2} \\
1 \frac{1}{2} \\
1 \frac{1}{2}\end{array}$ & $\begin{array}{l}20 \cdot 5 \\
22 \cdot 3 \\
13 \cdot 5 \\
17 \cdot 9 \\
11 \cdot 6\end{array}$ & $\begin{array}{l}18 \\
31 \\
15 \\
16 \\
11\end{array}$ & $\begin{array}{r}830 \\
1,016 \\
794 \\
1,010 \\
650\end{array}$ & $\begin{array}{l}4.05 \\
4.56 \\
5.86 \\
5.65 \\
5.58\end{array}$ & $\begin{array}{l}14.2 \\
18.0 \\
21.4 \\
25.8 \\
18.4\end{array}$ & $\begin{array}{l}0.151 \\
0.071 \\
0 \cdot 110 \\
0 \cdot 137 \\
0.083\end{array}$ & $\begin{array}{l}3.13 \\
2.76 \\
3.47 \\
1.83 \\
3.48\end{array}$ \\
\hline
\end{tabular}

Group I, one in Group II, and one in Group V. Fig. 2 shows the distribution of the ratio compliance

F.R.C.

comparison with a group of 50 normal subjects (Marshall, 1957). In Group I there is a tendency to low compliance figures, but the compliance in the other groups is distributed throughout the normal range.

The non-elastic resistance in a series of 50 normal subjects has been found to lie between 1.20 and $3.40 \mathrm{~cm}$. water per litre per second measured at a flow rate of 0.5 litres per second in those 37 subjects in whom the F.R.C. was 2.5 litres or more. In the 13 in whom the F.R.C. was below 2.5 litres there was a considerable increase in airway resistance with decreasing F.R.C. The number of normal subjects tested with F.R.C.s below 2.5 litres was too small to define accurate limits of normality, but if an "envelope" is drawn to enclose all the normal values (Fig. 3 ) the values in only three patients with sarcoidosis lie outside this envelope. In this figure conductance (reciprocal of resistance) has been plotted against F.R.C.
Two patients with abnormal values are in Group $\mathrm{I}$ and both of these patients have normal compliance ; the third patient is in Group V, also with normal compliance.

There is fair agreement between the E.F.R." and the M.B.C., but where these two values differ $\frac{0}{3}$ widely (patients 5, 16, 18, and 26) the M.B.C. is the lower one, presumably due to an insufficient 5 effort on the patient's part. The results obtained $\frac{D}{0}$

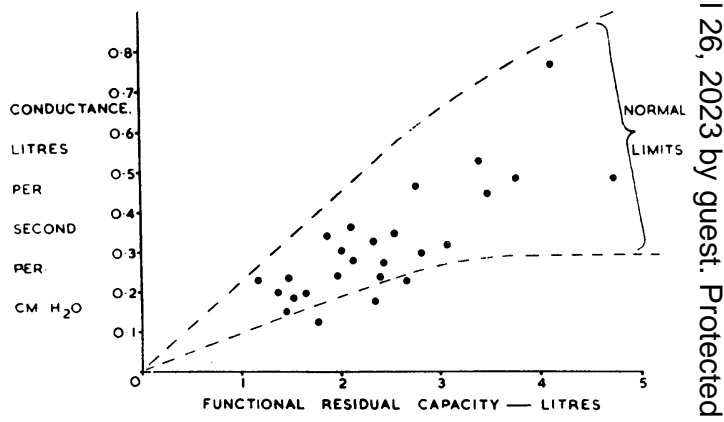

FIG. 3.-The relationship between lung conductance (reciprocal lofO non-elastic resistance) and F.R.C. The interrupted lines show the limits of normal values (Marshall, 1957). 
in these patients suggest that the E.F.R. ${ }^{40}$ test gives the more reliable estimate of expiratory obstruction. The assessment of ventilatory defect (based on the results of the E.F.R. ${ }^{40}$ test) shows impaired ventilation in some of the patients in all groups. Points of note, however, are the relative normality of patients in Group III (infiltration for less than two years) and the reduction in expiratory flow rates of patients in Group $V$ (enlarged hilar nodes). The assessment of ventilatory defect, based on the E.F.R. ${ }^{40}$, agrees fairly well with the values obtained for non-elastic resistance, but, since it does not take into account the F.R.C., the agreement is poor in some patients who have a low F.R.C. and in whom the non-elastic resistance is considered normal.

\section{Discussion}

The principal disturbance of pulmonary function that has been found in these patients is a defect of diffusion. Other abnormalities such as a ventilatory defect and reduced compliance were encountered, but are of minor importance.

The fact that pulmonary sarcoidosis may not be a disabling condition in spite of marked radiographic changes is supported by the findings in

TABLE IV

ASSESSMENT OF PULMONARY FUNCTION IN 26 PATIENTS WITH PULMONARY SARCOIDOSIS

\begin{tabular}{|c|c|c|c|c|c|}
\hline \multirow[b]{2}{*}{$\begin{array}{l}\text { Patient } \\
\text { No. }\end{array}$} & \multicolumn{2}{|c|}{ Dyspnoea Assessment } & \multirow{2}{*}{$\begin{array}{l}\text { Ventila- } \\
\text { tory } \\
\text { Defect }\end{array}$} & \multirow[b]{2}{*}{$\begin{array}{c}\text { Diffusion } \\
\text { Defect }\end{array}$} & \multirow{2}{*}{$\begin{array}{c}\text { Com- } \\
\text { pliance } \\
\text { Defect }\end{array}$} \\
\hline & Clinical & $\begin{array}{l}\text { Experi- } \\
\text { mental }\end{array}$ & & & \\
\hline $\begin{array}{c}\text { Group I } \\
1 \\
2 \\
3 \\
4 \\
5 \\
6 \\
\end{array}$ & $\begin{array}{l}2 \\
2 \\
1 \\
3 \\
1 \\
1 \\
\end{array}$ & $\begin{array}{l}0 \\
0 \\
1 \\
2 \\
0 \\
1\end{array}$ & $\begin{array}{l}0 \\
0 \\
0 \\
3 \\
1 \\
0\end{array}$ & $\begin{array}{l}0 \\
0 \\
2 \\
3 \\
2 \\
3 \\
\end{array}$ & $\begin{array}{l}0 \\
0 \\
1 \\
0 \\
0 \\
1\end{array}$ \\
\hline $\begin{array}{c}\text { Group II } \\
7 \\
8 \\
9\end{array}$ & $\begin{array}{l}3 \\
1 \\
0\end{array}$ & $\begin{array}{l}2 \\
0 \\
0\end{array}$ & $\begin{array}{l}2 \\
2 \\
1\end{array}$ & $\begin{array}{l}3 \\
1 \\
2\end{array}$ & $\begin{array}{l}1 \\
0 \\
0\end{array}$ \\
\hline $\begin{aligned} & \text { Group } I I J \\
& 10 \\
& 11 \\
& 12 \\
& 13 \\
& 14 \\
& 15\end{aligned}$ & $\begin{array}{l}0 \\
0 \\
1 \\
0 \\
0 \\
0\end{array}$ & $\begin{array}{l}0 \\
0 \\
0 \\
0 \\
0 \\
0\end{array}$ & $\begin{array}{l}0 \\
0 \\
0 \\
0 \\
0 \\
1\end{array}$ & $\begin{array}{l}\mathbf{0} \\
\mathbf{0} \\
\mathbf{0} \\
0 \\
2 \\
0\end{array}$ & $\begin{array}{l}0 \\
0 \\
0 \\
0 \\
0 \\
0\end{array}$ \\
\hline $\begin{array}{c}\text { Group } \\
16 \\
17 \\
17 \\
18 \\
19 \\
20 \\
21\end{array}$ & $\begin{array}{l}2 \\
0 \\
0 \\
1 \\
0 \\
0\end{array}$ & $\begin{array}{l}2 \\
0 \\
0 \\
0 \\
0 \\
0\end{array}$ & $\begin{array}{l}0 \\
0 \\
0 \\
1 \\
0 \\
0\end{array}$ & $\begin{array}{l}2 \\
2 \\
1 \\
1 \\
0 \\
.1\end{array}$ & $\begin{array}{l}0 \\
0 \\
0 \\
0 \\
0 \\
0\end{array}$ \\
\hline $\begin{array}{c}\text { Group } V \\
22 \\
23 \\
24 \\
25 \\
26\end{array}$ & $\begin{array}{l}1 \\
0 \\
0 \\
0 \\
0\end{array}$ & $\begin{array}{l}1 \\
2 \\
0 \\
0 \\
0\end{array}$ & $\begin{array}{l}2 \\
2 \\
1 \\
0 \\
0\end{array}$ & $\begin{array}{l}2 \\
0 \\
0 \\
0 \\
0\end{array}$ & $\begin{array}{l}\mathbf{0} \\
0 \\
1 \\
0 \\
0\end{array}$ \\
\hline
\end{tabular}

$0=$ Normal. $1=$ Slight impairment. $2=$ Moderate impairment. $3=$ Severe impairment. these patients. A few patients with very severe dyspnoea were excluded from this study, but of the 26 investigated only five had moderate or severe dyspnoea as assessed from their history and only four as assessed on the basis of treadmill exercise (Table IV). Of the five patients with dyspnoea of grade 2 or 3 as assessed clinically, two appeared to have no appreciable dyspnoea during treadmill exercise, and in these two (patients 1 and 2) the function tests showed no abnormality. A diffusion defect was found in nine of 12 patients who gave a history of dyspnoea and in six of seven in whom dyspnoea was observed on treadmill exercise.

From the overall assessment of function shown in Table IV it is clear that patients in Groups I and II have the most severely impaired function, the mean of the points allotted on assessment of Groups I and II being 2.7 and 4 per patient respectively, whilst in Groups III, IV, and V it is only $0.5,1.3$, and 1.6 respectively.

In order to relate disturbance of pulmonary function to the radiographic appearances in the lungs the latter may be divided in our 26 patients into four categories, namely, advanced fibrosis (Fig. 4), diffuse fine stippling (shadows measuring approximately $1 \mathrm{~mm}$. or less in diameter) (Fig. 5), diffuse reticulo-nodular shadows, measuring several millimetres in diameter (Fig. 6), and clear, including those with enlarged hilar nodes. The most severe defects of function occur in those

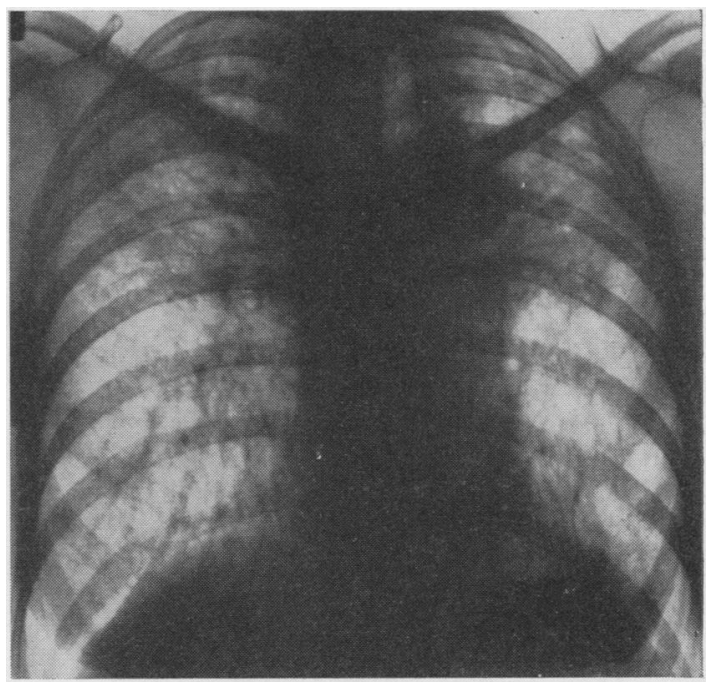

FIG. 4.-Chest radiograph of a woman aged 45 years (patient No. 4), showing advanced and fibrotic pulmonary sarcoidosis of 10 years' duration. The patient also had splenomegaly and a chronic uveitis. The diagnosis was supported histologically by liver and conjunctival biopsy. 


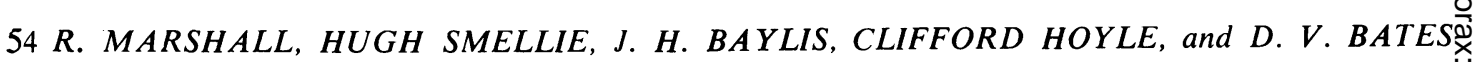

patients with advanced fibrosis, though patients 1 and 2 are exceptions. The pattern of fibrosis in those two patients is quite typical (Fig. 7) but of lesser extent than the others. Severe diffusion defect most commonly occurs in patients with diffuse fine stippling (patients 6,7 , and 14). We found that function is as impaired in patients whose lung fields have cleared as in those with diffuse reticulo-nodular shadows. This emphasizes that there is no close relationship between the radiographic appearances and pul-

FIG. 5.-Radiograph of part of the right lung field of a woman aged 39 years (patient No. 7), showing diffuse fine stippling in pulmonary sarcoidosis. The patient also had splenomegaly and enlarged peripheral lymph nodes. The diagnosis was supported histologically by liver biopsy.

FIG. 6.-Radiograph of part of the right lung field of a man aged 35 years (patient No. 8), showing diffuse reticulo-nodular shadows in pulmonary sarcoidosis. The patient also had sarcoidosis of the skin.

Fig. 7.-Chest radiograph of a woman aged 45 years (patient No. 2), with sarcoidosis of eight years' duration, showing pulmonary fibrosis to a lesser extent than that shown in Fig. 4 and associated with normal pulmonary function.

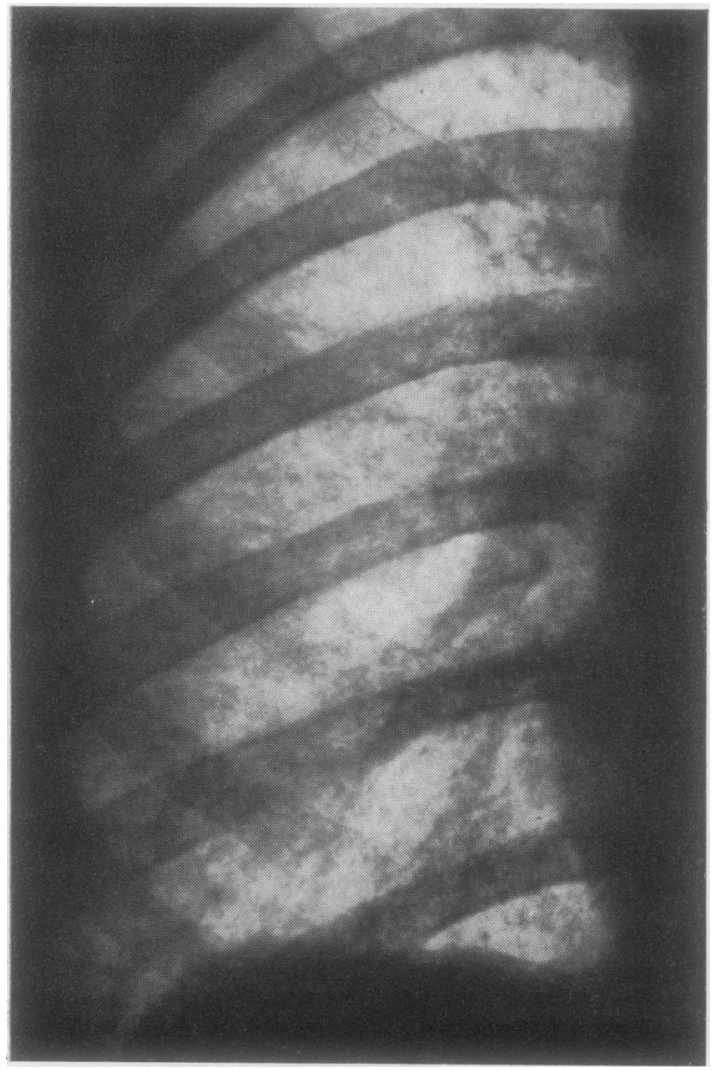

Fio. 5

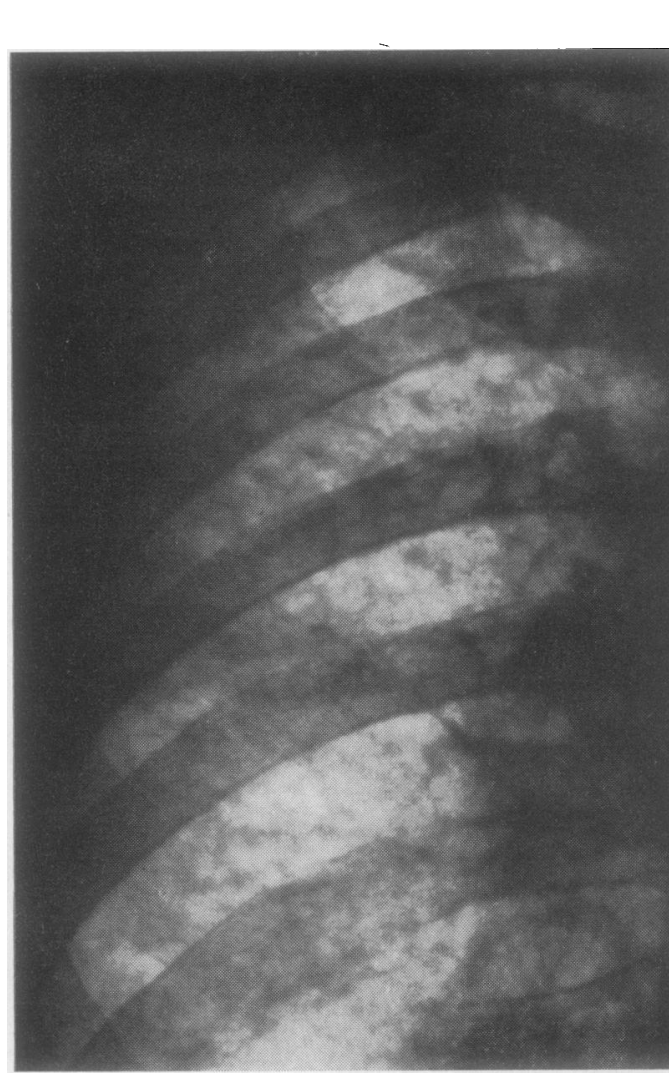

FIG. 6

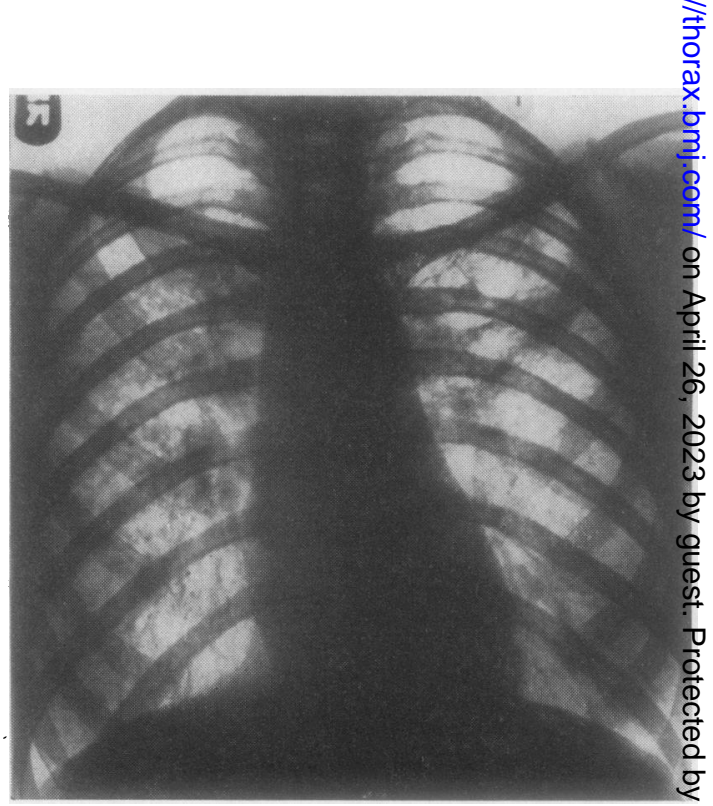

Fig. 7 


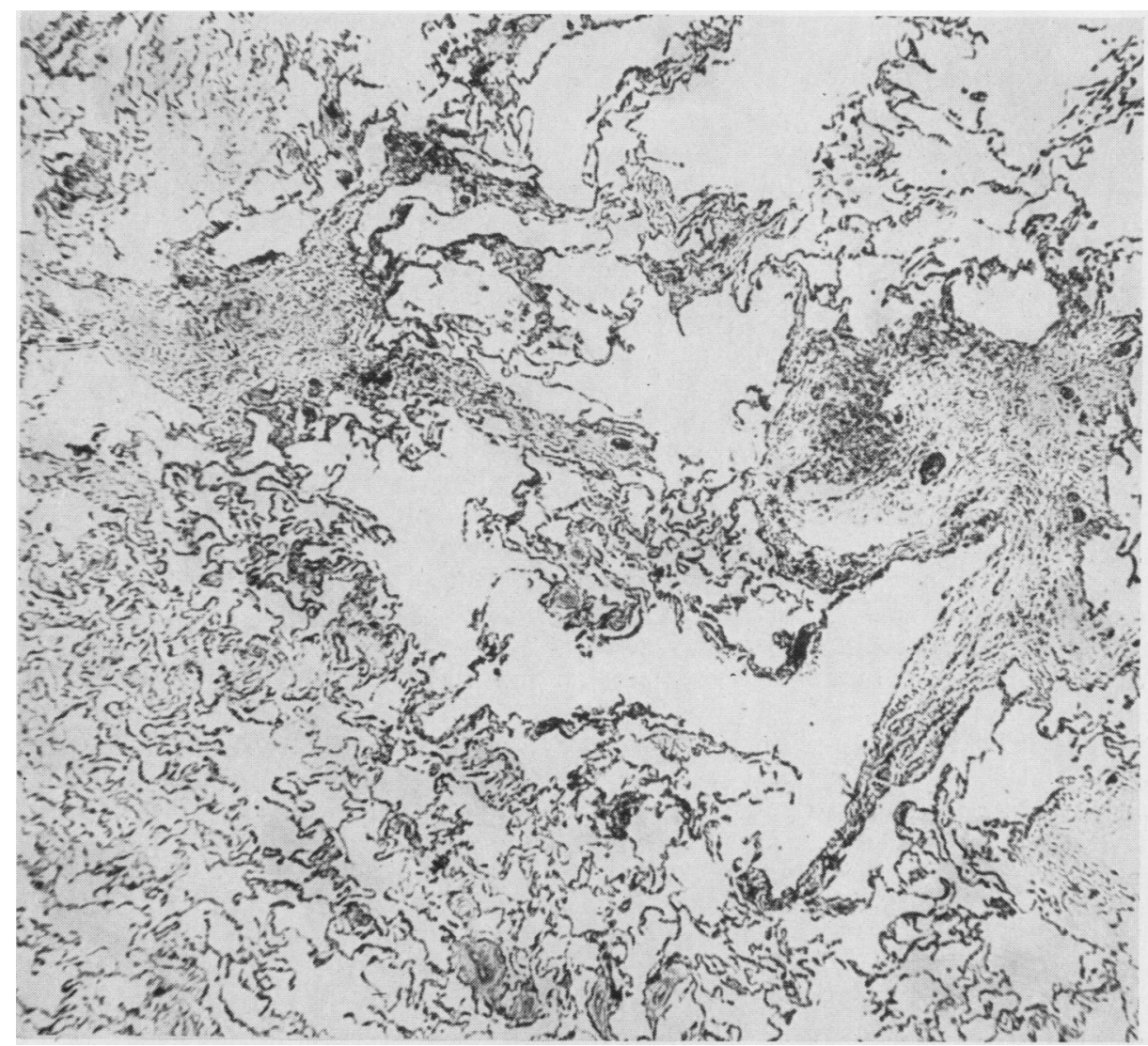

Fig. 8. - Section of a lung biopsy taken from a woman aged 45 years (patient No. 16), showing areas of fibrosis (haematoxylin and eosin, $\times$ 50). The patient presented with erythema nodosum and hilar node enlargement with bilateral coarse miliary shadows in the chest radiograph. The biopsy was taken one year later at a time when the chest radiograph had returned to normal.

monary function in sarcoidosis, a discrepancy which may be caused by the presence of subradiographic granulomata or fibrosis (Fig. 8). In estimating the effects of such changes we have related the duration of the disease in the clinical groups II, III, and IV (patients who have all had at some time diffuse mottling in the lung fields) to the degree of diffusion defect. The mean duration of disease in patients in these groups with diffusion defect is 44 months, while the mean for those with normal diffusion is 21 months, and there is a significant correlation between duration of disease and degree of diffusion defect as graded in Table IV $(r=+0.695$; S.E. $= \pm 0.27)$. It also seems from this that the advent of radiographic clearing, as occurred in Group IV, is of little value in assessing the prognosis in regard to function, especially pulmonary gas diffusion.

Some previous investigators have emphasized the tendency of patients with sarcoidosis to develop chronic obstructive emphysema. The largest reported series studied by means of function tests has been that of Stone, Schwartz, Feltman, and Lovelock (1953). They divided their patients into three groups based on the duration of the disease. They found that in the group (seven patients) with pulmonary involvement lasting for more than three years the type of functional abnormality could be either of the "emphysema" pattern with respiratory obstruction (three patients) or of the fibrosis pattern with predominant restriction of lung movement (three patients). One patient in this group had essentially normal pulmonary function tests. McClement, Renzetti, Himmelstein, and Cournand (1953) studied 10 patients and on the basis of pulmonary function tests divided them into three groups. The first group had evidence of reduced lung volume only, the second group reduced lung volumes and evidence of alveolar-capillary block 
(diffusion defect), and the third group (one patient only) pulmonary function tests suggestive of chronic obstructive emphysema. Coates and Comroe (1951) also reported findings suggestive of emphysematous changes in the five patients with pulmonary sarcoidosis whom they studied, as also was the case in the patient reported by Lukas (1951). On the other hand, Baldwin, Cournand, and Richards (1949) in their study of pulmonary fibrosis included five patients with sarcoidosis and specifically excluded any patients with ventilatory obstruction or raised residual air percentage. The diagnosis of emphysema in sarcoidosis has been based on increased residual volume, increased residual volume/total lung capacity ratio, impaired mixing efficiency, and spirographic evidence of respiratory obstruction. These criteria are often considered diagnostic of chronic obstructive emphysema such as occurs as the end-result of long-standing chronic bronchitis, but they could well be indicative of bronchial constriction alone without the development of loss of tissue and the tendency to bronchial collapse usually present in emphysema. The ratio of residual volume to total lung capacity so often used as an indication of emphysema is particularly misleading in a disease like sarcoidosis in which the reduction in total lung capacity alone can increase this ratio above the level at which it is often considered

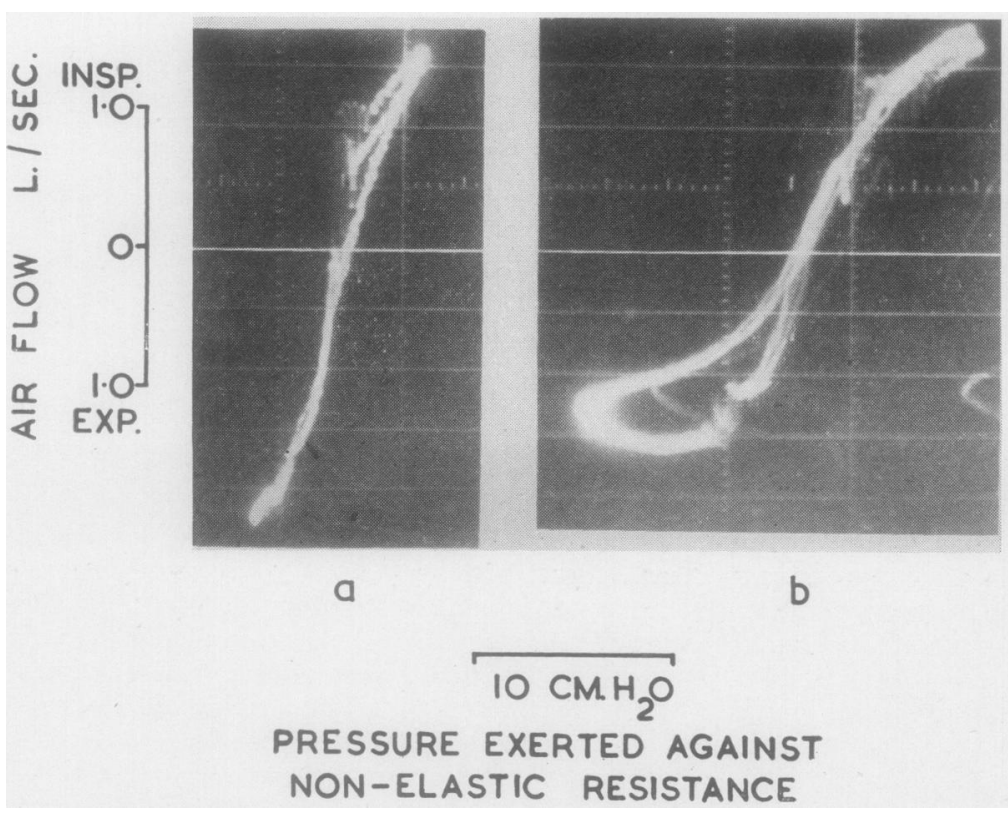

FIG. 9.-Tracings of flow and non-elastic pressure obtained by the method of Mead and Whittenberger (1953). The subjects were panting to produce high rates of air flow: (a) patient with sarcoidosis, $(b)$ patient with emphysema. diagnostic of emphysema. Only two of $\overrightarrow{\vec{A}_{\mathrm{e}}}$ patients studied here had a residual volume greaEr than one coefficient of variation more than the p dicted, but nine patients had a residual volums/ total lung capacity ratio greater than $35 \%$ e figure often taken to indicate emphysema). (8) the two patients with an increased residual volume, neither had a non-elastic resistance greater than $2.5 \mathrm{~cm}$. water per litre per second or a mixing efficiency less than $50 \%$. Moreover, the traci画g (Fig. 9) of air flow and non-elastic pressutwe obtained on the oscilloscope screen by the method of Mead and Whittenberger was not looped in the expiratory phase, as is found in patients with emphysema (Mead, Lindgren, and Gaensle्gr, 1955). Although the pulmonary function tests in the patients studied here show no evidence $\overrightarrow{a f}$ chronic obstructive emphysema, this does not clude the presence of localized areas of bullogis. emphysema which are often demonstrated histologically in sarcoidosis (Mallory, 1948 ; Longcoge and Freiman, 1952).

Many previous investigators have demonstrat the reduction in vital capacity and lung volums in sarcoidosis (Leitner, 1946; Galdston, Weise⿳亠口冋feld, Benjamin, and Rosenbluth, 1951; Bruce a rod Wassén, 1940 ; Small, 1951 ; Riley, Riley, and H霆, 1952 ; Austrian, McClement, Renzetti, Dona Riley, and Cournand, 1951 ; Williams, 1953), aßd some have reported a close association between changes in vital capacity and other lung function tests and the radiographic changes clinical findings (Bruce a Wassén, 1940 ; Small, 195\$; Lukas, 1951; Riley and others, 1952). If diminutiōn of vital capacity or inspirstory capacity were due changes in the lung ome might expect that the diminintion would be accompanied by abnormal stiffness of the lungs. In our patients there is a significant correlation between compliance ard inspiratory capacity $\left(\mathrm{r} \frac{\bar{Q}}{\mathrm{D}}\right.$ $+0.738 ;$ S.E. $= \pm 0.2$ ), but $\stackrel{?}{\text { of }}$ the seven patients with a sip nificantly low vital capacidy (less than $80 \%$ of predicte six have a compliance with normal limits taking in account the patient's F.R.\&. 
This suggests that the reduction of vital capacity in sarcoidosis is due more to the replacement of gas-containing lung by solid tissue than it is to increased stiffness of the lung tissue that is expanding. On the other hand, the reduction in diffusing capacity does not appear to be related to the reduction in lung volumes and the diffusing capacity is found to be impaired in many patients even when assessed in relation to the reduced F.R.C. Thus the effect of sarcoidosis is not only that of producing a "lobectomy"; the defect of function is not solely due to the reduction in the volume of functioning lung.

Several investigators have attempted to account for different patterns of impaired pulmonary function in sarcoidosis on the basis of different patterns of pulmonary fibrosis. The peribronchial pattern of fibrosis is thought to give rise to emphysema (Coates and Comroe, 1951; Stone and others, 1953 ; Spain, 1950), the interstitial type to alveolar capillary block (Austrian and others, 1951), and perivascular fibrosis to impaired diffusion and increased venous admixture (Riley and others, 1952). Our results show that ventilation, diffusion, and compliance are often affected simultaneously, but there is an isolated instance of increased non-elastic resistance without any other defect (patient 23), and in five patients an impaired diffusing capacity was found with no other, or out of proportion to any other, defect (patients $6,9,14,16$, and 17). In fact, of the three assessments-ventilation, diffusion, and compliancethe diffusion defect is the one most often seen in these patients, and change in compliance is least often seen. The relatively normal values for compliance are interesting in view of the degree of lung involvement seen radiographically. Patients in Group I with radiographic evidence of fibrosis most commonly show decreased compliance, but even in them the compliance may still be normal. On the other hand, the compliance may be significantly reduced in patients with apparently normal lung fields (patient 24).

The normal values for compliance in relation to the F.R.C. suggest that, although the sarcoid lesion has reduced the volume of expanding lung, the elastic properties of the remaining alveoli have not altered and expansion therefore occurs in the relatively normal tissue between the granulomata. In some of the patients the diffusing capacity in relation to the F.R.C. is moderately or severely impaired in spite of a normal compliance. If the functioning alveoli were thickened one would expect a decrease in compliance to accompany the impaired diffusing capacity, as happens, for instance, in the alveolar fibrosis of the HammanRich syndrome. Histological examination of the lungs in sarcoidosis (Schaumann, 1936 ; Mallory, 1948) shows that the granulomata may have a perivascular distribution and may also invade and obliterate blood vessels; this focal obstruction to perfusion of the lung could cause the reduced diffusing capacity. Five out of the six patients in Group IV in whom the lung lesions cleared radiographically were left with impaired diffusion.

In order to provide more definite evidence of the relationship of diffusing capacity to progress of the disease it is necessary to carry out repeated tests of diffusing capacity during the different stages of the disease. Riley and others (1952) found no significant increase of the diffusing capacity for oxygen in three patients after A.C.T.H. treatment although the lesions partially cleared in two. McClement and others (1953) reported an increase of the oxygen-diffusing capacity in two out of five patients treated with cortisone. It is possible that vessels involved in the early stages of granulomata may have their function restored when the granulomata regress, but vessels which are more seriously involved are obliterated completely by fibrous tissue.

The oxygen utilization $\left(\mathrm{O}_{2} / \mathrm{V}\right.$, the oxygen uptake in ml. per litre of ventilation) in only four subjects falls below the range of values $(5.42 \pm 1.5)$ found by Donald, Bishop, Cumming, and Wade (1955) for normal subjects exercising to approximately the same degree. In their subjects there

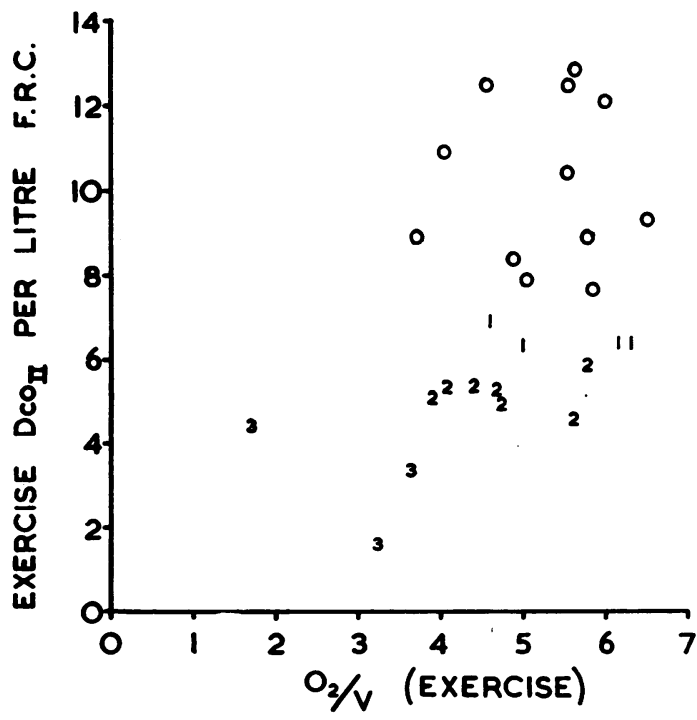

Fig. 10.-The relationship between Doo per litre F.R.C. and the $\mathrm{O}_{2} / \mathrm{V}$ ratio on exercise. The numerals $0,1,2$, and 3 indicate the asesssment of the diffusion defect. 
was no variation in the oxygen utilization on exercise with oxygen uptakes between about 300 and $1,000 \mathrm{ml}$. per minute. The oxygen uptake in the patients investigated here varied from 390 to $1,600 \mathrm{ml}$. per minute (mean $920 \mathrm{ml}$. per minute). Of the four patients with low oxygen utilization three were in Group I and one in Group II. If the ventilation in these patients is determined by the need to maintain an adequate oxygen uptake, then the oxygen utilization might be expected to correlate with the diffusing capacity of the lungs. In Fig. 10 the exercise $D_{c o}$ per unit volume of F.R.C. is plotted against the $\mathrm{O}_{2} / \mathrm{V}$, both measurements being made on exercise. This shows a general relationship between the two observations, the correlation coefficient being significant at the $2 \%$ level $(r=+0.476)$. The work of Ogilvie, Forster, Blakemore, and Morton (1957) has shown that the diffusing capacity, as measured by a single breath technique, is proportional to body surface area. In this study, the correlation coefficient between $D_{\text {co }}$ per unit body surface area and oxygen utilization is +0.644 (S.E. \pm 0.2 ). These observations certainly suggest a relationship between the diffusing capacity and the level of ventilation required for a given oxygen uptake.

We conclude from this investigation that the main lesion of pulmonary function in sarcoidosis is a reduction in diffusing capacity of the lungs. In addition, reduction of the lung volume by the disease may contribute to the disability by impairing the mechanical functions of the lung. Long-standing disease is accompanied by greater impairment of diffusion and possibly by decreased compliance when fibrosis occurs. Radiographic clearing of the lesions may not be accompanied by a return of normal function. No close relationship exists between the radiographic appearances of the lungs and the defects in pulmonary function, but patients with diffuse fine stippling or reticulation were more often found to have a lowered diffusing capacity, and radiographic evidence of fibrosis was sometimes accompanied by decreased compliance. As far as can be determined from this study, in which patients at different stages of the disease were examined on one occasion only, detailed pulmonary function tests are capable of indicating the effect of the present and past lesions on the performance of the lungs. Measurements of lung volumes and ventilatory defect alone give little useful information.

\section{SUMMARY}

The lung volumes, mixing efficiency, mechanical properties of the lungs, and diffusing capacity were measured in 26 patients with pulmonary sar- coidosis. The patients were selected to represent $\overrightarrow{\vec{s}}$ all stages of the disorder and each was allocated $\overrightarrow{0}$ to one of five groups according to the duration of $\frac{C}{0}$ the disease and the radiographic appearances of $\frac{}{\omega}$ the lungs. The results of the pulmonary function $\widehat{\varnothing}$ tests are discussed in relation to these groups.

No evidence of obstructive emphysema was found and the compliance of the lungs was usually $\vec{O}$ normal. The commonest lesion of pulmonary $\vec{\overrightarrow{ }}$ function was a diffusion defect which occurred ${ }_{\sigma}$ more frequently with long-standing disease. Five $\overrightarrow{\vec{z}}$ out of six patients in whom pulmonary sarcoidosis ${ }^{x}$ had cleared radiographically at the time of the $\vec{\omega}$ tests showed impaired diffusion.

We are indebted to all the patients in this series for $\stackrel{\circ}{?}$ their willing co-operation and to many colleagues $\vec{Z}$ who referred them to the care of one of us (C. H.);@ to Professor H. A. Magnus for his generous help withơ the histology of biopsy specimens; and to Mr. L. S. Bartlett and Miss J. A. West for technical assistance. 0 Some of our expenses were defrayed by a grant fromo King's College Hospital Research Fund.

\section{REFERENCES}

Austrian, R., McClement, J. H., Renzetti, A. D., Donald, K. W. Riley, R. L., and Cournand, A. (1951). Amer. J. Med., 11, 667 .O Baldwin, E. deF., Cournand, A., and Richards, D. W. (jr.) (1949) Medicine (Baltimore), 28, 1.

Bates, D. V., Boucot, N. G., and Dormer, A. E. (1955). J. Physiol: (Lond.), 129, 237.

and Christie, R. V. (1950). Clin. Sci., 9, 17

Bruce, T., and Wassén, E. (1940). Acta med. scand., 104, 63.

Coates, E. O., and Comroe, J. H. (jr.) (1951). J. clin. Invest., 30, 848

Comroe, J. H. (jr.), Forster, R. E., DuBois, A. B., Briscoe, W. A. and Carlsen, E. (1955). The Lung. Year Book Publishers Chicago. Donald, K. W., Bishop, J. M., Cumming, G., and Wade, O. L. (1955)
Clin. Sci., 14, 37.

Dornhorst, A. C., and Kelly, H. B. (1954). Lancet, 1, 290.

Galdston, M., Weisenfeld, S., Benjamin, B., and Rosenbluth, M. B (1951). Amer. J. Med., 10, 166.

Kennedy, M. C. S. (1953). Thorax, 8, 73.

Leitner, S. J. (1946). Cardiologia (Basel), 10, 379.

Longcope, W. T., and Freiman, D. G. (1952). Medicine (Baltimore), $31,1$.

Lukas, D. S. (1951). Amer. Rev. Tuberc., 64, 279.

McClement, J. H., Renzetti, A. D., Himmelstein, A., and Cournand A. (1953). Amer. Rev. Tuberc., 67, 154.

Mallory, T. B. (1948). Radiology, 51, 468.

Marshall, R. (1957). Clin. Sci., 16, 507.

- and DuBois, A. B. (1956). Ibid., 15, 473

Mead, J., Lindgren, I., and Gaensler, E. A. (1955). 34, J., Lindgren, I., and Gaensler, E. A. (1955). J. clin. Invest. N

- and Whittenberger, J. L. (1953). J. appl. Physiol., 5, 779.

Needham, C. D., Rogan, M. C., and McDonald, I. (1954). Thorax, $9,313$.

Ogilvie, C. M., Forster, R. E., Blakemore, W. S., and Morton, J. W (1957). J. clin. Invest., 36, 1.

Pappenheimer, J. R., Comroe, J. H., Cournand, A., Ferguson $₫$ J. K. W., Filley, G. F., Fowler, W. S., Gray, J. S.,' Helmholtz, , H. T., Ötis, A. B., Răhn, H., and Riley, R. L. (1950). Fed: Proc., 9, 602.

Riley, R. L., Riley, M. C., and Hill, H. M. (1952). Bull. Johns Hopk Hosp., 91, 345 .

Schaumann, J. (1936). Brit. J. Derm., 48, 399.

Small, M. J. (1951). J. Amer. med. Ass., 147, 932.

Spain, D. M. (1950). Ann. intern. Med., 33, 1150.

Stone, D. J., Schwartz, A., Feltman, J. A., and Lovelock, F. J. (1953) Amer. J. Med., 15, 468.

Williams, M. H. (jr.) (1953). J. clin. Invest., 32, 909. 